\title{
NEWMAN'S THEOREM IN THE RIEMANNIAN CATEGORY
}

\section{BY}

\author{
L. N. MANN (1) AND J. L. SICKS
}

\begin{abstract}
In $1931 \mathrm{M}$. H. A. Newman proved that if $M$ is a connected topological manifold with metric $d$, there exists a number $\epsilon>0$, depending only upon $M$ and $d$, such that every compact Lie group $G$ acting effectively on $M$ has at least one orbit of diameter at least $\epsilon$. Aside from isolated results nothing appears to be known about $\epsilon$. In order to learn more about the invariant $\epsilon$, attention is restricted here to groups of isometries on a Riemannian manifold. It is found that the invariant $\epsilon$ of $M$ is connected with the notion of convexity introduced by J. H. C. Whitehead in 1932 .
\end{abstract}

1. Introduction. In $1931 \mathrm{M}$. H. A. Newman proved the following result. Theorem (Newman) [7], [2]). If $M$ is a connected topological manifold with metric $d$, there exists a number $\epsilon>0$, depending only upon $M$ and $d$, such that every compact Lie group $G$ acting effectively on $M$ has at least one orbit of diameter at least $\epsilon$.

Later P. A. Smith [8] , [1, III.9] gave a generalization of Newman's theorem to cohomology manifolds with fixed coverings. The several proofs of Newman's theorem do not shed much light on the nature of the invariant $\epsilon$. If $M$ is the unit sphere $S^{n-1}$ with the standard metric in $R^{n}$ and if we restrict our attention to orthogonal effective actions of $G$ on $S^{n-1}$, then it can be shown that $\epsilon=$ $2 \pi / 3$ is best possible. However, aside from isolated results like this, nothing appears to be known about $\epsilon$.

In order to learn more about the invariant $\epsilon$, a good starting place appears to be the Riemannian category; in other words we restrict our attention to groups of isometries on a Riemannian manifold $M$. We have found that here the invariant $\epsilon$ of $M$ is connected with the notion of convexity introduced by Whitehead in [9]. For each $p \in M$ we introduce a positive number $r_{p}$ called the radius of convexity of $M$ at $p$ (compare with [3]). If $K$ is a compact subset of $M, \inf _{K} r_{p}>0$. Our results are easily stated for the case when $M$ is compact.

Received by the editors July $26,1974$.

AMS (MOS) subject classifications (1970). Primary 57E10, 53C20; Secondary 54H15.

Key words and phrases. Newman's theorem on periodic transformations, groups of isometries, diameter of orbits, radius of convexity.

(1) The author was partially supported by NSF Grant GP-29664.

Copyright $\odot$ 1975, American Mathematical Society 
THEOREM (TheOREMS 2 AND 4). Suppose $M$ is a compact Riemannian manifold with $r(M)=\inf _{p \in M} r_{p}$. If $G$ is a compact Lie group of isometries acting effectively on $M$, there exists at least one orbit of diameter at least $r(M)$. In fact, if $G$ acts without fixed points, every orbit has diameter at least $r(M)$.

If $M$ is not compact, our results are incomplete for the fixed point case as we cannot deal in an entirely satisfactory way with the situation where the isometry group $G$ is a finite group of odd order (Theorem 3 ).

The techniques of this paper were suggested to us by [6]. The authors are grateful to Professor Hans Samelson for his valuable suggestions and to their colleagues H. T. Ku and David Hoffman for the proof of Theorem 4.

2. Preliminaries and the fixed point free case. Let $M$ be a Riemannian manifold with metric $d(-,-)$.

Definition. A subset $S$ of $M$ will be called very strongly convex if the following hold:

(1) For all $x, y \in S$, there exists a unique geodesic $g_{x y}$ in $S$ joining $x$ to $y$, and length $g_{x y}=d(x, y)$.

(2) Moreover $g_{x y}$ is the only geodesic in $M$ joining $x$ to $y$ having length equal to $d(x, y)$.

(3) If $g_{u v}$ is a geodesic in $S$ joining $u$ to $v$ and $q$ is any point of $S, d(q, z)$ $<\operatorname{Max}\{d(q, u), d(q, v)\}$ for each interior point $z$ of $g_{u v}$.

Our notion of very strongly convex was suggested by the stark konvex of [3, p. 159]. Recall that $S$ is called stark konvex if it and all open balls contained in it satisfy condition (1). It is easy to verify that very strongly convex implies stark konvex. We shall say more about this relationship later.

PROPOSITION 1. Let $S$ be a compact very strongly convex subset of $M$. If $G$ is a compact Lie group of isometries acting effectively on $M$ and with no fixed points in $S$, then $S$ contains no orbit of the action.

PRoOF. If $S$ contains an orbit, it must contain some orbit, say $G(a)$, of minimum diameter. Since $G(a)$ is not a fixed point, there exists $c \in G(a), c \neq a$. Let $g_{a c}$ be the unique geodesic in $S$ joining $a$ to $c$ with length $g_{a c}=d(a, c)$. Let $b$ be any interior point of $g_{a c}$. We show that

(i) $G(b) \subset S$, and

(ii) diam $G(b)<\operatorname{diam} G(a)$,

which is, of course, a contradiction.

(i) Let $\lambda \in G$. Then $\lambda g_{a c}$ is a geodesic joining $\lambda a$ to $\lambda c$ where $\lambda a, \lambda c \in$ $G(a) \subset S$. Now

$$
\text { length } \lambda g_{a c}=\text { length } g_{a c}=d(a, c)=d(\lambda a, \lambda c) \text {. }
$$


So by condition (2) of very strong convexity, $\lambda g_{a c}$ must be the unique geodesic in $S$ joining $\lambda a$ to $\lambda c$. Consequently $\lambda b \in \lambda g_{a c} \subset S$ and $G(b) \subset S$ since $\lambda$ was an arbitrary element of $G$.

(ii) Choose $\mu \in G$ such that $d(\mu b, b)=\operatorname{diam} G(b)$. Consider $\mu b$ and the geodesic $g_{a c}$ in $S$. By condition (3) of very strong convexity,

(A) $d(\mu b, b)<\operatorname{Max}\{d(\mu b, a), d(\mu b, c)\}$. Considering $c$ and $\mu g_{a c}$ in $S$,

(B) $d(c, \mu b)<\operatorname{Max}\{d(c, \mu a), d(c, \mu c)\} \leqslant \operatorname{diam} G(a)$ and considering $a$ and $\mu g_{a c}$ in $S$,

(C) $d(a, \mu b)<\operatorname{Max}\{d(a, \mu a), d(a, \mu c)\} \leqslant \operatorname{diam} G(a)$. So by (A), (B), and (C),

$$
\operatorname{diam} G(b)=d(\mu b, b)<\operatorname{diam} G(a) .
$$

For $p \in M$ and $\rho>0$, let

$$
B(p, \rho)=\{x \in M / d(x, p)<\rho\}
$$

denote the open ball of radius $\rho$ about $p$.

DEFINITION. The radius of convexity of $M$ at a point $p$ is denoted by $r_{p}$ and defined as follows:

$r_{p}=\sup \{\rho / B(p, \rho)$ is very strongly convex and $\overline{B(p, \rho)}$ is compact $\}$.

REMARK. Alternatively

$$
r_{p}=\sup \{\rho / B(p, \rho) \text { is very strongly convex and compact }\} .
$$

In general, our radius of convexity is smaller than the Konvexitätsradius of [3, p. 162]. The Konvexitätsradius of $M$ at $p$ is denoted by $r_{p}^{*}$ and defined as follows:

$$
r_{p}^{*}=\sup \{\rho / B(p, \rho) \text { is stark konvex }\} .
$$

If $S^{n-1}$ is the unit sphere with the standard metric in $R^{n}$,

$$
\begin{gathered}
r_{p}^{*}=r_{p}=\pi / 2 \text { for } n=2, \\
r_{p}^{*}=\pi / 2, r_{p}=\pi / 4 \text { for } n>2 .
\end{gathered}
$$

We have the following comparison between $r_{p}$ and $r_{p}^{*}$.

PROPOSITION 2. $r_{p}^{*} / 3 \leqslant r_{p} \leqslant r_{p}^{*}$ where $r_{p}^{*}$ is the Gromoll-Klingenberg-Meyer "Konvexitätsradius" of $M$ at $p$.

Proof. Since a very strongly convex ball is stark konvex, the right-hand inequality is immediate.

Let $0<\rho<r_{p}^{*}$. It is sufficient to show that $B_{0}=B(p, \rho / 3)$ is very strongly 
convex. Clearly $B_{0}$ satisfies condition (2) of very strong convexity and $\bar{B}_{0}$ is compact. So suppose $g_{u v}$ is a geodesic in $B_{0}$ from $u$ to $v$, and $q$ a point of $B_{0}$ such that there exists an interior point $z$ of $g_{u v}$ with

$$
d(q, z)=\max _{w \in g_{u v}} d(q, w) .
$$

Let $h(t)$ be the unique geodesic in ' $B_{0}$ from $z$ to $q$ and choose $q^{\prime}$ pn $h(t)$ slightly past $q$. Now

$$
d\left(q^{\prime}, z\right)=d\left(q^{\prime} q\right)+d(q, z) \geqslant d\left(q^{\prime}, q\right)+d(q, u)
$$

and $d\left(q^{\prime} q\right)+d(q, u)>d\left(q^{\prime}, u\right)$ since, otherwise, the unique geodesic from $q^{\prime}$ to $u$ would have to coincide with $-h(t)$ from $q^{\prime}$ to $q$ and, consequently, from $q^{\prime}$ to $z$ which is clearly absurd. So $d\left(q^{\prime}, z\right)>d\left(q^{\prime}, u\right)$ and similarly, $d\left(q^{\prime}, z\right)>d\left(q^{\prime}, v\right)$. Finally choose $z^{\prime}$ on $g_{u v}$ such that

$$
d\left(q^{\prime}, z^{\prime}\right)=\max _{w \in g_{u v}} d\left(q^{\prime}, w\right)
$$

Now $g_{u v}$ does not lie in the open ball around $q^{\prime}$ with radius $d\left(q^{\prime}, z^{\prime}\right)$. However, this is a contradiction since this ball and any slightly larger ball (which necessarily contains $g_{u v}$ ) are stark konvex.

COROLlaRY. (1) $r_{p}>0$ for all $p \in M$.

(2) If $r_{p_{0}}=+\infty$ for some $p_{0} \in M$, then $r_{p}=+\infty$ for all $p \in M$.

(3) $r_{p}$ is a continuous function of $p$ in $M$.

Proof. (1) and (2) follow immediately since $r_{p}^{*}$ has these properties [3]. The continuity of $r_{p}$ may be shown in exactly the same way as the continuity of $r_{p}^{*}$ is shown in $[3, \mathrm{p} .162]$.

THEOREM 1. Let $M$ be a Riemannian manifold. Then if $G$ is a compact Lie group of isometries acting effectively and with no fixed points on $M$, $\operatorname{diam} G(p) \geqslant r_{p}$, each $p \in M$. In particular if there exists $p \in M$ with $r_{p}=+\infty$, every compact Lie group of isometries acting on $M$ must have a fixed point.

Proof. Let $\gamma$ be any number such that $0<\gamma<r_{p}$. Then $\overline{B(p, \gamma)}$ is a compact very strongly convex subset of $M$. By Proposition 1 , diam $G(p)>\gamma$.

REMARK. The last part of Theorem 1 implies a result of E. Cartan [4, p. 75].

THEOREM 2. Let $M$ be a Riemannian manifold. Define

$$
r(M)=\inf _{p \in M} r_{p}, \quad R(M)=\sup _{p \in M} r_{p} .
$$

Then if $G$ is a compact Lie group of isometries acting effectively and with no fixed points on $M$, we have the following: 
(1) Every orbit has diameter at least $r(M)$.

(2) If $\widetilde{R}$ is any number such that $0<\widetilde{R}<R(M)$, there exists at least one orbit of diameter at least $\widetilde{R}$.

In particular if $r(M)=+\infty$, every compact Lie group of isometries acting on $M$ must have a fixed point.

PRoof. Theorem 2 is, of course, an immediate consequence of Theorem 1.

EXAMPLE. If $M$ is the unit sphere $S^{n-1}$ with the standard metric, $r(M)=$ $R(M)=\pi / 4 \quad(n \geqslant 3)$. However, as we noted in the Introduction, $\epsilon=2 \pi / 3$ is best possible in this case. In this case we obtain a better bound from Theorem 5 .

We call a Riemannian manifold $M$ homogeneous if the group of isometries of $M$ acts transitively on $M$. If $M$ is homogeneous, it is easy to verify that $r_{p}$ is a fixed positive constant (possibly $+\infty$ ) for all $p \in M$. Consequently,

REMARK. If $M$ is either compact or homogeneous, then $r(M)>0$.

\section{Fixed points.}

THEOREM 3. Suppose $M$ is a Riemannian manifold. Let $\widetilde{R}$ be any number, $0<\widetilde{R}<R(M)$. Suppose $G$ is a compact Lie group of isometries acting effectively (and possibly with fixed points) on $M$. Then if either

(1) $G$ is not a finite group of odd order, or

(2) $M$ has nonpositive sectional curvatures, there exists at least one orbit of diameter larger than $\widetilde{R} / 2$.

Proof. Choose $p \in M$ such that $r_{p}>\widetilde{R}$. Then $\overline{B(p, \widetilde{R} / 2)}$ is a compact very strongly convex subset of $M$. If $\overline{B(p, \widetilde{R} / 2)}$ contains no fixed points of the action, it follows directly from Proposition 1 that diam $G(p)>\widetilde{R} / 2$.

Suppose then $f \in \overline{B(p, \widetilde{R} / 2)}$ with $G(f)=f$. Then $\overline{B(f, \widetilde{R} / 2)}$ is stark konvex. Since $f$ is a fixed point of $G, G$ acts as a group of differentials and, in fact, orthogonally and effectively on the tangent space $M_{f}$. Let

$$
S^{*}=S(0, \widetilde{R} / 2)=\left\{x^{*} \in M_{f} / d^{*}\left(x^{*}, 0\right)=\widetilde{R} / 2\right\}
$$

be the sphere of radius $\widetilde{R} / 2$ about 0 in $M_{f}$, where $d^{*}(-,-)$ is the euclidean metric on $M_{f}$. Since $\overline{B(p, \widetilde{R} / 2)}$ is stark konvex, the exponential map, exp, is a diffeomorphism of $S^{*}=S(0, \widetilde{R} / 2)$ onto

$$
S=S(f, \widetilde{R} / 2)=\{x \in M / d(x, f)=\widetilde{R} / 2\} .
$$

Moreover exp is equivariant with respect to the actions of $G$ on $S^{*}$ and $S$.

Case (1) $G$ is not a finite group of odd order. We restrict our attention to a subgroup $H$ of $G$ with $H \cong Z_{2}$. The action of $G$, and hence $H$, is orthogonal and effective on $S^{*}$ so there exists an orbit of $H$ consisting of $\left\{x_{1}^{*},-x_{1}^{*}\right\}$, i.e., 
a pair of antipodal points of $S^{*}$. Now $d^{*}\left(x_{1}^{*},-x_{1}^{*}\right)=\widetilde{R}$. Let $x_{1}=\exp x_{1}^{*}, y_{1}$ $=\exp \left(-x_{1}^{*}\right)$. So $x_{1}, y_{1} \in S$. Since exp is equivariant, $\left\{x_{1}, y_{1}\right\}$ is an orbit of $H$ on $S$. Moreover $d\left(x_{1}, y_{1}\right)=\widetilde{R}$, since the unique geodesic joining $x_{1}$ to $y_{1}$ is the image under exp of the straight line segment through $x_{1}^{*}, 0$ and $-x_{1}^{*}$ in $M_{f}$. Hence

$$
\operatorname{diam} G\left(x_{1}\right) \geqslant \operatorname{diam} H\left(x_{1}\right)=\widetilde{R}>\widetilde{R} / 2 .
$$

Case (2) $M$ has nonpositive sectional curvatures. We may assume $G$ is finite of odd order and restrict our attention to a subgroup $H$ of $G$ with $H \cong Z_{p}$, $p$ an odd prime. Since $H$ acts orthogonally on $S^{*}, H$ acts invariantly and effectively on some great circle $C^{*}$ of $S^{*}$. It is easy to verify that an orbit of $H$ on $C^{*}$ has diameter

$$
\widetilde{R} \sin \left[\left(\frac{p-1}{p}\right) \pi / 2\right] \geqslant \widetilde{R} \sin \left[\frac{2}{3} \cdot \frac{\pi}{2}\right]=\frac{\sqrt{3}}{2} \widetilde{R} .
$$

So let $x_{1}^{*}, y_{1}^{*} \in C^{*}$ be on $H\left(x_{1}^{*}\right)$ with

$$
d^{*}\left(x_{1}^{*}, y_{1}^{*}\right)=\operatorname{diam} H\left(x_{1}^{*}\right) \geqslant \frac{\sqrt{3}}{2} \widetilde{R} .
$$

Then $x_{1}=\exp x_{1}^{*}, y_{1}=\exp y_{1}^{*}$ are on $H\left(x_{1}\right) \subset S \subset M$. Since $M$ has nonpositive sectional curvatures, it follows $[4$, p. 73$]$ that

$$
d\left(\exp x_{1}^{*}, \exp y_{1}^{*}\right) \geqslant d^{*}\left(x_{1}^{*}, y_{1}^{*}\right)
$$

So

$$
\operatorname{diam} G\left(x_{1}\right) \geqslant \operatorname{diam} H\left(x_{1}\right) \geqslant d\left(x_{1}, y_{1}\right) \geqslant d^{*}\left(x_{1}^{*}, y_{1}^{*}\right) \geqslant \frac{\sqrt{3}}{2} \widetilde{R}>\widetilde{R} / 2 .
$$

REMARK. It is likely that Theorem 3 can be extended to the case where the sectional curvatures of $M$ are bounded. In any event, if $M$ is compact we have the following complete result.

THEOREM 4. Suppose $M$ is a compact Riemannian manifold. Then if $G$ is a compact Lie group of isometries acting effectively (and possibly with fixed points) on $M$, there exists at least one orbit of diameter at least $r(M)$.

Proof. If the fixed point set $F$ is empty, the result follows immediately from Theorem 2. Choose $p \in M$ such that

$$
d(p, F)=\max _{x \in M} d(x, F)=l .
$$

We shall show that $r_{p} \leqslant l$ which, using Proposition 1, implies that diam $G(p) \geqslant$ $r_{p} \geqslant r(M)$. We suppose then that $r_{p}>l$ and reach a contradiction. There exists $f \in F$ with $d(p, f)=d(p, F)$. Now $f$ must be unique for suppose there exists $f^{\prime} \in F, f^{\prime} \neq f$ with $d\left(p, f^{\prime}\right)=d(p, f)=l<r_{p}$. Let $l<\rho<r_{p}$ and $B_{0}=B(p, \rho)$. 
Then $B_{0}$ is very strongly convex. Let $g_{f f^{\prime}}$ be the unique geodesic in $B_{0}$ joining $f$ to $f^{\prime}$. As $F$ is totally geodesic, $g_{f f^{\prime}} \subset F$. Therefore for any interior point $u$ of $g_{f f^{\prime}}$,

$$
d(p, u) \geqslant \min _{v \in F} d(p, v)=d(p, f)=d\left(p, f^{\prime}\right) .
$$

But this contradicts the fact that $B_{0}$ is very strongly convex. Hence $f$ is unique.

Let $h(t)$ be the unique geodesic in $B_{0}$ from $f$ to $p, h\left(t_{0}\right)=p$. Take a sequence $p_{n}$ on $h(t)$ past $p$ and converging to $p$. Let $f_{n}$ be a point of $F$ with $d\left(p_{n}, f_{n}\right)=d\left(p_{n}, F\right)$ and note that $d\left(p_{n}, f_{n}\right) \leqslant d(p, f)$. Since $f$ is the unique point of $F$ with $d(p, F)=d(p, f)$, a subsequence of the $f_{n}$ 's converge to $f$. Consider $G(t, z)=d(h(t), z)$ in a small neighborhood of $\left(t_{0}, f\right)$. Since we are within $B_{0}$ and $G$ stays away from zero, $G$ is a $C^{\infty}$ function of $t$ and $z$ [5, p. 166]. Clearly $\partial G / \partial t>0$ at $\left(t_{0}, f\right)$ and, consequently, in a small enough neighborhood of $\left(t_{0}, f\right)$. It follows that for some $n, d\left(p_{n}, f_{n}\right)>d\left(p, f_{n}\right)>d(p, f)$. So we have a contradiction and therefore it must be that $r_{p} \leqslant l$.

We used our notion of radius of convexity rather than that of [3] since it simplified our proofs and gave us a very concise statement of Theorem 1. It is of course possible to state all our results in terms of the Gromoll-KlingenbergMeyer "Konvexitätsradius" using the left-hand inequality of Proposition 2. However, in some cases, sharper results may be obtained by direct arguments. For example we can show the following.

THEOREM 5. Suppose $M$ is a compact Riemannian manifold with $r^{*}(M)=$ $\inf _{p \in M} r_{p}^{*}$. If $G$ is a compact Lie group of isometries acting effectively on $M$, there exists at least one orbit of diameter at least $r^{*}(M)$. In fact, if $G$ acts without fixed points, every orbit has diameter at least $2 r^{*}(M) / 3$.

ADDED IN PROOF. Karsten Grove and Hermann Karcher [Math. Z. 132 (1973)] have investigated $C^{1}$-close group actions on compact Riemannian manifolds and have obtained in this setting several Newman type results.

\section{REFERENCES}

1. Glen E. Bredon, Introduction to compact transformation groups, Academic Press, New York, 1972.

2. Andreas Dress, Newman's theorems on transformation groups, Topology 8 (1969), 203-207. MR 38 \#6629.

3. D. Gromoll, W. Klingenberg and W. Meyer, Riemannsche Geometrie im Grossen, Lecture Notes in Math., no. 55, Springer-Verlag, Berlin and New York, 1968. MR 37 \#4751.

4. S. Helgason, Differential geometry and symmetric spaces, Pure and Appl. Math., vol. 12, Academic Press, New York and London, 1962. MR 26 \#2986.

5. S. Kobayashi and K. Nomizu, Foundations of differential geometry. Vol. 1, Interscience, New York and London, 1963. MR 27 \#2945. 
6. Deane Montgomery and Leo Zippin, A theorem on Lie groups, Bull. Amer. Math. Soc. 48 (1942) 448-452. MR 4, 3.

7. M. H. A. Newman, $A$ theorem on periodic transformations of spaces, Quart. J. Math. 2 (1931), 1 -9.

8. P. A. Smith, Transformations of finite period. III. Newman's theorem, Ann. of Math. (2) 42 (1941), 446-458. MR 2, 324.

9. J. H. C. Whitehead, Convex regions in the geometry of paths, Quart. J. Math. 3 (1932), 33-42.

DEPARTMENT OF MATHEMATICS, UNIVERSITY OF MASSACHUSETTS, AMHERST, MASSACHUSETTS 01002 